\title{
Intranasal Delivery of Nano Neurotherapeutics: A Meta Opinion
}

\author{
Chandrakantsing V Pardeshi ${ }^{1}$, Abhijeet D Kulkarni ${ }^{1}$, Veena S Belgamwar ${ }^{2}$ and Sanjay J Surana ${ }^{3}$ \\ ${ }^{1}$ Department of Pharmaceutics, R. C. Patel Institute of Pharmaceutical Education and Research, India \\ ${ }^{2}$ Department of Pharmaceutical Sciences, R.T.M. Nagpur University, India \\ ${ }^{3}$ Department of Pharmacognosy, R. C. Patel Institute of Pharmaceutical Education and Research, India
}

Submission: February 17, 2017; Published: March 15, 2017

*Corresponding author: Chandrakantsing V Pardeshi, Assistant Professor, Department of Pharmaceutics,R. C. Patel Institute of Pharmaceutical Education and Research, India, Tel: +91 9881414752; Fax: +91 2563 251808; Email: chandrakantpardeshi11@gmail.com

\begin{abstract}
The most stringer barrier for the delivery of neurotherapeutics in-vivo is presented by the blood-brain barrier which limits the access of neurotherapeutics to the CNS effectively. Intranasal delivery of neurotherapeutics is one of the promising endeavors to surmount the blood-brain barrier, designated as direct nose to brain transport, by the passage of neurotherapeutics along the olfactory and trigeminal nerve pathways. The outstanding findings obtained from the nanomedicines in the preclinical studies for the treatment of CNS disorders via intranasal administration have harnessed the scientists from various research groups to walk around the nano neurotherapeutics for the effective management of CNS disorders. Currently, 33 nanotherapeutics and 67 commercialized nanodevices are on the market but none of them is a nano neurotherapeutic. This could be attributed to the fact that though the researchers have obtained outstanding preclinical findings but these were followed by the disappointing clinical results. To conclude in summary, the authors want to mention here that the development of targets-specific nano pharmaceuticals delivered via non-invasive intranasal route of administration might present some hope to the development of novel neurotherapeutics for major CNS disorders. According to our assessment, in order to come out with a newly designed neurotherapeutic with optimized physicochemical, pharmacological, and pharmacokinetic properties, it requires several parameters to be studied in silico, in-vitro, and in-vivo, keeping in mind the complex nature of the brain.
\end{abstract}

Keywords: Nanotechnology; Nanomedicine; Neurotherapeutics; Intranasal; Drug delivery; CNS disorders; Nose to brain

Abbreviations: NIH: National Institute of Health; CNS: Central Nervous System; CSF: Cerebrospinal Fluid; nm: Nanometers; PLGA: Poly (lacticco-Glycolic Acid)

\section{Introduction}

Nanotechnology is a multidisciplinary field where Nano refers to the scale of objects measured in nanometers (nm) means one nanometer is one billionth, or $10^{-9}$, of a meter. The dimensions of nanoparticles are similar to biomolecules, such as proteins $(1-20 \mathrm{~nm})$, DNA $(\sim$ diameter $2 \mathrm{~nm})$, virus $(\sim 20$ $\mathrm{nm})$, cell surface receptors $(\sim 10 \mathrm{~nm})$, hemoglobin $(\sim 5 \mathrm{~nm})$, cell membrane ( $\sim 6-10 \mathrm{~nm})$. Therefore scientists with diverse interests and backgrounds have clutched their attention to work with and understand properties of materials on a nano scale and apply them in medicine [1]. Nanomedicine comes along one of the most important disciplines of nanotechnology and according to National Institute of Health (NIH), the term nanomedicine refers to highly specific medical intervention at the molecular scale for diagnosis, prevention and treatment of various diseases $[2,3]$.
National Nanotechnology Initiative defines nanotechnology as the production of materials in the scale between 1 and 100 billionths of a meter (1-100 nm) at least in one dimension [4].

The last 20 years have witnessed an outburst in research on the development of novel drug delivery systems. Among them, the multiparticulate drug delivery systems have broad prospects in the pharmaceutical field [5]. Researchers from various disciplines have been harnessed by the superior outcomes obtained from such nanocarrier systems viz. greater therapeutic efficacy and reduced dosing frequency [6]. At the University of North Carolina, a chemistry Professor Joseph De Simone once said about nanoparticles that you want to deliver it where you want it, when you want it, without wasting it [7]. A very first report was published on the designing a specified drug 
delivery system to achieve selective targeting of drugs and was originated from the perception of Paul Ehrlich, who proposed a hypothesis of magic bullet concept [8]. Neurotherapeutics represents second largest segments of the total drug market accounting for a total market share of US $\$ 56$ billion, and will be growing substantially in the recent years, majorly because of the rapidly increasing population with CNS disorders. However, neurotherapeutics takes longer time to be there in the market. This is attributed to the rigorous restrictions set down by the physiological barriers in the CNS [9-11].

Drug delivery via nasal route has been practices since ancient times for systemic effects. Nasal drug delivery has now been recognised as an excellent platform for the delivery of neurotherapeutics. Large surface area, porous endothelial membrane, high total blood flow, ready accessibility, and avoidance of hepatic first pass metabolism are few of the major reasons for drug delivery across nasal mucosa. Henceforth, the intranasal delivery of neurotherapeutics is attaining the great deal of attention of the researchers, now-a-days $[12,13]$.

Animal and human investigations have proved that, transport of exogenous materials directly from nose to brain is a potential route for bypassing the blood-brain barrier [14,15]. Nose to brain drug delivery is most likely mediated along the olfactory and/or trigeminal nerve pathways, located at the roof of the nasal cavity as its neuroepithelium is the only part of the CNS that is directly exposed to the external environment. Thus, better targeting action can be achieved due to direct movement of drug from the submucosal space of the nose into the cerebrospinal fluid (CSF) compartment of the brain [16,17]. Again, the novel nasal administration devices have proven their proficiency in the delivery of neurotherapeutics or nano neurotherapeutics so as to achieve the site-specific action. The performance of the nasal administration device depends on three major attributed viz. quality, safety and efficacy [18].

The most attractive area of research in drug delivery, now-adays, is the design of nanocarriers for targeted drug therapy. In author's opinion, the nanotechnology-mediated delivery of CNS acting drugs (in general designated as nano neurotherapeutics) via intranasal route of drug administration is the topic of great attention in the today's research. Extensive clinical efforts are still needed so as to bring the nano neurotherapeutics to the shelves for the well being of society.

\section{Intranasal Delivery of Nano Neurotherapeutics: Research at a Glance}

There is ample of literature is available demonstrating the extensive investigations published on the theme nanotechnologybased delivery of neurotherapeutics via intranasal route of administration. The nanoparticulate formulations have been developed and evaluated for the brain targeting ability of neurotherapeutics in the treatment of variety of CNS disorders [19]. Our research group is investigating the potential of nanotechnology-based nose to brain drug delivery systems in the management of various CNS disorders since last decade. The major nanoparticulate formulations we have developed in our laboratory include the solid lipid nanoparticles [20], and polymer-lipid hybrid nanoparticles [21], polyelectrolyte nanocomplex [22,23], and N,N,N-trimethyl chitosan-based mucoadhesive nanoparticles [24] for the management of Parkinson's disease, and PLGA-based nanoparticles [25] for the treatment of Schizophrenia.

The intranasal delivery of nano neurotherapeutics has also been explored globally for various other nano formulations viz. chitosan nanoparticles of venlafaxin [26], estradiol [27], and ropinirole [28], nano emulsion of zonisamide [29], risperidone $[30,31]$, olanzapine [32,33], polymeric micelles of zolmitriptan [34], micro emulsion of tacrine [35], and so on.

\section{Conclusion and Future Perspectives}

The CNS disorders (mainly brain cancers, neurodegenerative disorders and cerebrovascular disorders) have threatened the human health with high mortality rates. According to the WHO statistics, CNS disorders constitute about $12 \%$ of total deaths globally. Thus, the field of development of neurotherapeutics for the treatment of CNS disorders is growing rapidly in the pharmaceutical sector, now-a-days. However, poor permeability and distribution of neurotherapeutics within the CNS led to the little clinical success of the neurotherapeutics as an effective treatment strategy for CNS disorders. There a number of strategies available to enhance the brain targeting ability of neurotherapeutics and intranasal drug delivery is one of the promising avenue to unlock the limitations posed by the other routes of drug administration or other formulation strategies. In general, the trend for the effective brain targeting of neurotherapeutics follows the below-mentioned sequence:

Nasal mucoadhesive nanoparticles > Nasal solution > Intravenous nanoparticles > Intravenous solution

Nano technological interventions could be able to improve the brain targeting ability of neurotherapeutics. Thereby, nano neurotherapeutics would be useful to reduce the gap between preclinical and clinical investigations, to increase the success rate of neurotherapeutics in clinical studies and further, their scaleup and commercialization aspects. The current findings indicate that the development of nano neurotherapeutics will certainly occupy the pharmaceutical market, the only query is when? To conclude in summary, the authors want to mention here that the development of targets-specific nano pharmaceuticals delivered via intranasal route of administration might present some hope to the development of novel neurotherapeutics for major CNS disorders. In order to come out with a newly designed neurotherapeutic with optimized physicochemical, pharmacological, and pharmacokinetic properties, it requires several parameters to be studied in silico, In-vitro, and In-vivo, 


\section{Global Journal of Nanomedicine}

keeping in mind the complex nature of the brain.

\section{Declaration of Interest}

The authors have no relevant affiliations or financial involvement with any organization or entity with a financial interest in or financial conflict with the subject matter or materials discussed in the manuscript. This includes employment, consultancies, honoraria, stock ownership or options, expert testimony, grants or patents received or pending, or royalties.

\section{References}

1. Patra CR, Bhattacharya R, Mukhopadhyay D, Mukherjee P (2010) Fabrication of gold nanoparticles for targeted therapy in pancreatic cancer. Adv Drug Deliv Rev 62(3): 346-361.

2. National Institutes of Health (2006) National Institute of Health Roadmap for Medical Research: Nanomedicine.

3. Webster TJ (2006) Nanomedicine: What's in a definition? Int J Nanomedicine 1(2): 115-116.

4. http://www.nano.gov.

5. Pardeshi C, Rajput P, Belgamwar V, Tekade A, Patil G, et al. (2012) Solid lipid based nanocarriers: an overview. Acta Pharma 62(4): 433-472.

6. Pardeshi CV, Rajput PV, Belgamwar VS, Tekade AR (2012) Formulation, optimization and evaluation of spray-dried mucoadhesive microspheres as intranasal carriers for valsartan. J Microencapsul 29(2): 103-114

7. http://www.scientificamerican.com/article.cfm?id=size-shapematter-nanotech-drug.

8. Patel S, Patel B, Patel Z, Pardeshi C (2012) Nanocarriers as Novel Noseto-Brain Targeted Drug Delivery Platforms. Indian Journal of Novel Drug Delivery 4: 243-251.

9. Alvijeh MS,ChishtyM,QaiserMZ,PalmerAM(2005)Drugmetabolismand pharmacokinetics, the blood-brain barrier, and central nervous system drug discovery. NeuroRx 2(4): 554-571.

10. http://www.wikinvest.com/wiki/Central_Nervous_System_Drug_ Market

11. Kulkarni AD, Patel HM, Surana SJ, Belgamwar VS, Pardeshi CV (2016) Brain-blood ratio: implications in brain drug delivery. Expert Opin Drug Deliv 13(1): 85-92.

12. Mistry A, Stolnik S, Illum L (2009) Nanoparticles for direct nose-tobrain delivery of drugs. Int J Pharm 379(1): 146-157.

13. Patel Z, Patel B, Patel S, Pardeshi C (2012). Nose to brain targeted drug delivery bypassing the blood-brain barrier: an overview. Drug Invention Today 4: 610-615.

14. Pardeshi CV, Belgamwar VS (2013) Direct nose to brain drug delivery via integrated nerve pathways bypassing the blood-brain barrier: an excellent platform for brain targeting. Expert Opin Drug Deliv 10(7): 957-972.

15. Abhijeet D Kulkarnia, Deepak B Baria, Sanjay JSuranab, Chandrakantsing V Pardeshi (2016) In-vitro, ex-vivo and in-vivo performance of chitosan-based spray-dried nasal mucoadhesive microspheres of diltiazem hydrochloride. Journal of Drug Delivery Science and Technology, 31: 108-117.

16. Ali J, Ali M, Baboota S, Sahani JK, Ramassamy C (2010) Potential of nanoparticulate drug delivery systems by intranasal administration Curr Pharm Des 16(14): 1644-1653.
17. Alam MI, Beg S, Samad A, Baboota S, Kohli K, et al. (2010) Strategy for effective brain drug delivery. Eur J Pharm Sci 40(5): 385-403.

18. Pardeshi CV, Vanjari YH, Kulkarni AD (2015) Novel nasal devices for the efficient drug delivery: a systemic review. Indian Journal of Novel Drug Delivery, 7: 1-9.

19. Kulkarni AD, Vanjari YH, Sancheti KH, Belgamwar VS, Surana SJ, et al. (2015) Nanotechnology-mediated nose to brain drug delivery for Parkinson's disease: A mini review. J Drug Target 23(9): 775-788.

20. Pardeshi CV, Rajput PV, Belgamwar VS, Tekade AR, Surana SJ (2013) Novel surface-modified solid lipid nanoparticles as intranasal carriers for ropinirole hydrochloride: application of factorial design approach. Drug Deliv 20(1): 47-56.

21. Pardeshi CV, Belgamwar VS, Tekade AR, Surana SJ (2013) Novel surface modified polymer-lipid hybrid nanoparticles as intranasal carriers for ropinirole hydrochloride: in-vitro, ex-vivo and in-vivo pharmacodynamic evaluation. J Mater Sci Mater Med 24(9): 21012115

22. Pardeshi CV, Belgamwar VS (2016) Ropinirole-Dextran Sulfate Nanoplex for Nasal Administration Against Parkinson's Disease: In Silico Molecular Modeling and in Vitro-Ex Vivo Evaluation. Artif Cells Nanomed Biotechnol 45 (3): 635-648.

23. Kulkarni AD, Vanjari YH, Sancheti KH, Patel HM, Belgamwar VS, et al. (2016) New nasal nanocomplex self-assembled from charged biomacromolecules: N,N,N-trimethyl chitosan and dextran sulfate. International Journal of Biological Macromolecules, 88: 476-490.

24. Pardeshi CV, Belgamwar VS (2016) Controlled synthesis of N,N,Ntrimethyl chitosan with modulated bioadhesion and nasal membrane permeability. Int J Biol Macromol 82: 933-944.

25. Shailesh S Chalikwar, Bhushan S Mene, Chandrakant V Pardeshi, Veena S Belgamwar, Sanjay J Surana (2013) Self-assembled chitosangrafted PLGA nanoparticles for intranasal delivery. Polymer-Plastics Technology and Engineering 52: 368-380.

26. Haque S, Md S, Fazil M, Kumar M, Sahni JK, et al. (2012) Venlafaxin loaded chitosan NPs for brain targeting: pharmacokinetic and pharmacodynamic evaluation. Carbohydr Polym 89(1): 72-79.

27. Wang X, Chi N, Tang X (2008) Preparation of estradiol chitosan nanoparticles for improving nasal absorption and brain targeting. Eur J Pharm Biopharm 70(3): 735-740.

28. Jafarieh O, Md S, Ali M, Baboota S, Sahni JK, et al. (2015) Design, characterization, and evaluation of intranasal delivery of ropiniroleloaded mucoadhesive nanoparticles for brain targeting. Drug Dev Ind Pharm 41(10): 1674-1681.

29. Shahiwala Aliasgar, Dash Dilip (2010) Preparation and evaluation of microemulsion based formulations for rapid-onset intranasal delivery of zonisamide. Advanced Science Letters, 3(4): 442-446.

30. Kumar M, Pathak K, Misra A (2009) Formulation and characterization of nanoemulsion-based drug delivery system of risperidone. Drug Development and Industrial Pharmacy, 35(4): 387-395.

31. Kumar M, Misra A, Babbar AK, Mishra AK, Mishra P, et al. (2008) Intranasal nanoemulsion based brain targeting drug delivery system of risperidone. Int J Pharm 358(1-2): 285-291.

32. Kumar M, Misra A, Mishra AK, Mishra P, Pathak K (2008) Mucoadhesive nanoemulsion-based intranasal drug delivery system of olanzapine for brain targeting. J Drug Target 16(10): 806-814.

33. SejuU,Kumar A,SawantKK(2011)Developmentandevaluation ofolanzapineloaded PLGA nanoparticles for nose-to-brain delivery: in vitro and in vivo studies. Acta Biomater 7(12): 4169-4176. 
34. Jain R, Nabar S, Dandekar P, Patravale V (2010) Micellar nanocarriers: potential nose-to-brain delivery of zolmitriptan as novel migraine therapy. Pharm Res 27(4): 655-664.
35. Jogani VV, Shah PJ, Mishra P, Mishra AK, Mishra AR (2008) Intranasal mucoadhesive microemulsion of tacrine to improve brain targeting. Alzheimer Dis Assoc Disord 22(2): 116-124.

Your next submission with JuniperPublishers
will reach you the below assets
- Quality Editorial service
- Swift Peer Review
- Reprints availability
- E-prints Service
- Manuscript Podcast for convenient understanding
- Global attainment for your research
- Manuscript accessibility in different formats
( Pdf, E-pub, Full Text, Audio)
- Unceasing customer service
Track the below URL for one-step submission
https://juniperpublishers.com/submit-manuscript.php

